\title{
Awake craniotomy for vestibular schwannoma. Is it a novel or a first report?
}

\author{
Klaus Novak ${ }^{1}$ (D) \\ Received: 13 February 2018 / Accepted: 19 February 2018 / Published online: 28 February 2018 \\ (C) Springer-Verlag GmbH Austria, part of Springer Nature 2018
}

Dear Editor,

With great interest I read the article of Shinoura et al. [4], reporting on their experience in the attempt to preserve hearing in microsurgery for vestibular schwannoma using awake craniotomy. The authors reported hearing preservation and useful facial nerve function in all of the patients in whom they have used intraoperative testing of the hearing level and facial nerve function. The intraoperative testing was performed by continuous communication and assessment of the patients by a neurosurgeon and neurologist. This is what appears to be a novel approach in the fight against cranial nerve morbidity in the surgery of vestibular schwannomas. The editorial, however, comments on the article as a first report of awake craniotomy in vestibular schwannoma surgery [1]. Awake craniotomy in the surgery for vestibular schwannoma was indeed used by the renowned Swedish neurosurgeon Herbert Olivecrona in the earlier cases of 415 tumors removed between 1931 and 1969 at the Karolinska Institute [3]. Awake craniotomy was used before general anesthesia became available at this institution. Olivecrona reported that incomplete tumor removal was associated with the cases of awake craniotomy, whereas after introduction of general anesthesia, incomplete removal was limited to cystic tumors [3]. This compares well to the article of Shinoura et al., who reported a high rate of incomplete tumor removal in their series of eight patients operated under local anesthesia, too. Intraoperative monitoring of facial nerve function was performed similar to the methods described in the work of Shinoura and co-authors: Olivecrona asked a nurse to observe the patient's face during the operation in frequent intervals. When the facial nerve was identified, its functionality was tested by electrical stimulation [2]. The authors Shinoura et al. do not mention that the study is the first report on awake craniotomy for vestibular schwannoma surgery [4]. A reference to previous experiences with awake surgery in the history of neurosurgery, however, would have been appropriate. In the editorial, the series of eight patients is presented as the first report of awake craniotomy in patients with vestibular schwannoma, which is clearly not correct if we explore the archives of medical history. One should be careful when calling a report a first!

\section{References}

1. Lund-Johansen M (2017) Awake craniotomy for vestibular schwannoma. Acta Neurochir 159:1587-1588

2. Nielsen A (1942) Acoustic tumors: with special reference to endresults and sparing of the facial nerve. Ann Surg 115(5):849-863

3. Olivecrona H (1967) Acoustic Tumours. J Neurosurg 26(1):6-13

4. Shinoura N, Midorikawa A, Hiromitsu K, Saito S, Yamada R (2017) Preservation of hearing following awake surgery via the retrosigmoid approach for vestibular schwannomas in eight consecutive patients. Acta Neurochir 159:1579-1585

This article is part of the Topical Collection on History of Neurosurgery

Klaus Novak

Klaus.novak@meduniwien.ac.at

1 Department of Neurosurgery, Medical University of Vienna, Währinger Gürtel 18-20, 1090 Wien, Austria 